\title{
Anabases
}

ANABASES Traditions et réceptions de l'Antiquité

$9 \mid 2009$

Varia

\section{Il carteggio Gaetano De Sanctis - Giuseppe Fraccaroli}

\section{Michele Curnis}

\section{(2) OpenEdition \\ 1 Journals}

Edizione digitale

URL: http://journals.openedition.org/anabases/530

DOI: 10.4000/anabases.530

ISSN: 2256-9421

\section{Editore}

E.R.A.S.M.E.

\section{Edizione cartacea}

Data di pubblicazione: 1 marzo 2009

Paginazione: 273-278

ISSN: 1774-4296

\section{Notizia bibliografica digitale}

Michele Curnis, « II carteggio Gaetano De Sanctis - Giuseppe Fraccaroli », Anabases [Online], 9| 2009, Messo online il 01 mars 2012, consultato il 21 octobre 2019. URL : http://journals.openedition.org/ anabases/530 ; DOI : 10.4000/anabases.530

Questo documento è stato generato automaticamente il 21 ottobre 2019.

(c) Anabases 


\title{
Il carteggio Gaetano De Sanctis - Giuseppe Fraccaroli
}

\author{
Michele Curnis
}

Marcella Guglielmo (ed.), Il carteggio Gaetano De Sanctis - Giuseppe Fraccaroli, Carteggi di Filologi, 7, diretti da Rosario Pintaudi, Università degli Studi di Messina, Dipartimento di Filologia e Linguistica, Edizioni Gonnelli, Firenze, 2007, 192 p., 50 euros, ISBN 978-88-89250-04-4.

1 Quando due protagonisti di tutt'un epoca culturale e politica, due personalità legate tra loro dalla scienza, dalla passione civile, dall'amicizia, oltre alla loro opera di studiosi e didatti lasciano anche corrispondenze private, i lettori delle generazioni a venire non possono che trarre materia, prove ulteriori e preziose, per confermare e argomentare meglio la grandezza e la civiltà degli interlocutori, in questo caso già ampiamente testimoniate. Gaetano De Sanctis e Giuseppe Fraccaroli sono, negli annali della storiografia e della filologia classica di fine Otto- e prima parte del Novecento, figure molto nitide, dagli intendimenti chiari e precisi. Storico di Roma per antonomasia, il primo ; fautore dell'irrazionale in letteratura, e dunque nemico del filologismo e delle tecniche ecdotiche ed esegetiche di provenienza germanica, il secondo. Nonostante la grande diversità di formazione, di prospettive e di indagine scientifica, De Sanctis e Fraccaroli svilupparono un sentimento reciproco di amicizia e di stima professionale tenace e costante - pur in anni molto difficili - nell'ambito dell'Università di Torino. L'Ateneo piemontese fu infatti il polo di attrazione per entrambi gli studiosi, dal momento che Fraccaroli, allievo a Padova di Eugenio Ferrai, vi fu chiamato nel 1895, per succedere a Giuseppe Müller sulla cattedra di Letteratura greca ; De Sanctis, allievo a Roma di Karl Julius Beloch, fu chiamato nel 1900 come Professore straordinario di Storia antica (e dal 1903 divenne ordinario). E nell'ambiente torinese « Alla scontrosità piemontese la scontrosità di De Sanctis non mai dispiacque. A Torino, negli anni precedenti alla prima guerra mondiale, passò gli anni più felici. C'erano tra i suoi colleghi uomini la cui stima De Sanctis poteva ricambiare senza sforzo. I nomi di Carlo Cipolla, di "Renier bonus" e soprattutto di Arturo Graf e di Giuseppe Fraccaroli non furono mai pronunciati da lui senza che la voce gli si facesse calda » (così Arnaldo Momigliano, In memoria di Gaetano De Sanctis [1957], ora in Secondo contributo alla storia 
degli studi classici, Roma 1960, p.301). Puntare l'attenzione su De Sanctis e la sua personalità, il suo stile di scrittura, le sue attenzioni e premure, significa individuare la sostanza del carteggio pubblicato da Marcella Guglielmo : pochi mesi dopo il suo arrivo a Torino è già testimoniata la corrispondenza con Fraccaroli, nei toni informali di amici che si conoscono molto bene. «Settantadue lettere indirizzate da Gaetano De Sanctis all'amico e collega Giuseppe Fraccaroli nel periodo compreso tra il 2 luglio 1901 e il 25 aprile 1917, poco più di un anno prima della morte di Fraccaroli, sono conservate presso la Biblioteca Civica di Verona. " Così prende avvio il paragrafo Una «corrispondenza scientifica» (p. 1), primo dell'Introduzione curata da Guglielmo, molto precisa nel porgere al lettore tutte le coordinate storiche, geografiche, sociali e biografiche in cui il carteggio si è sviluppato (gli altri paragrafi della stessa Introduzione, p. 1-27, hanno per titolo Fraccaroli, De Sanctis : il loro tempo, De Sanctis e Fraccaroli : il carteggio, La vita privata, Nel laboratorio dello studioso, De Sanctis e Fraccaroli Maestri, Le dispute, La cronaca e la storia del tempo). Segue una postilla (p. 31-33), esplicativa delle metodologie di lavoro d'archivio, della ricerca bibliografica e dell'indagine sui documenti originali, grazie alla quale la curatrice ha potuto stabilire criticamente una sequenza di pubblicazione: «Le lettere sono state trascritte seguendo l'ordine cronologico. Nonostante esse fossero già state numerate presso l'archivio della Biblioteca Civica di Verona, la contestualizzazione e il raffronto tra le singole carte ha consentito di inserire nella sequenza che appariva più verosimile alcune lettere non datate e di datare diversamente la prima, la cui intestazione era di incerta lettura; per questo ogni missiva è stata numerata con due numeri : il primo indica la successione cronologica qui proposta, il secondo, tra parentesi quadre, riproduce l'ordine delle carte manoscritte secondo la sistemazione veronese" (p.32). Il volume è quindi strutturato in modo da presentare le settantadue lettere di De Sanctis (p.35-127), corredate di note a piè di pagina di carattere esplicativo e storico-letterario. Delle risposte di Fraccaroli è stato possibile trascrivere e pubblicare soltanto una parte esigua (in nota alle lettere di De Sanctis cui fanno riferimento), poiché tutte le carte donate dallo studioso alla Biblioteca Civica di Verona sono sotto tutela dell'Istituto dell'Enciclopedia Italiana fondato da G. Treccani. La parte finale del libro è una succosa Appendice (p. 129-174) di documenti non inediti, ma giustamente ripubblicati, in quanto di difficile reperibilità e molto opportuni al completamento e alla esatta comprensione di numerose lettere precedenti di De Sanctis.

2 Al di là della narrazione dei singoli eventi, dei « fattori » biografici dei protagonisti, uno dei motivi di interesse del carteggio tra i due studiosi - che Guglielmo subito coglie e presenta in apertura dell'Introduzione - è la presenza di critiche e polemiche tra i corrispondenti, a proposito della rispettiva opera scientifica. Se De Sanctis espone, in forma privata e pubblica, tutte le sue perplessità a proposito dell'esegesi irrazionalistica dell'arte letteraria, di cui Fraccaroli diventa veemente fautore contro il metodo storico-critico di Vitelli e della sua scuola (oltre che, naturalmente, dei filologi d'oltralpe), Fraccaroli critica lo stile espositivo della Storia dei Romani, di cui corregge le prime bozze di stampa. Già Silvio Accame, nel curare la ristampa della Storia (vol. I, $3^{\text {a }}$ edizione, Firenze 1979), aveva studiato e pubblicato materiali inediti, contenenti le osservazioni del lettore d'eccezione; con il confronto delle lettere di De Sanctis il dialogo diventa più ricco e le osservazioni di Fraccaroli perspicue. E la curatrice del carteggio annota che « La loro corrispondenza privata consente di comprendere ora la reale portata del contributo di Fraccaroli : De Sanctis gli spedisce le pagine ed egli con "franco giudizio" (Lettera XXII [22]) risponde, ora segnalando con estrema precisione 
refusi e inesattezze, ora soffermandosi a esaminare passi di cui propone diverse soluzioni ; a sua volta altrettanto apertamente l'autore le mette poi in discussione nelle lettere di risposta. Frequente è il riferimento allo stile, che l'attento revisore rimprovera come "saltante e slegato" e scarsamente uniforme" (p. 12). Ciascuno manifesta dunque giudizio sincero e critico, senza ipocrisie e affettazione, poiché dietro tale giudizio resta sempre quel sentimento di autentica amicizia e affetto ricordato anche da Momigliano. La reazione ai pareri dell'amico non è mai piccata o superficiale ; ma possono farsi strada i consigli di un accomodamento intelligente, oppure quelli dell'ironia, per "non prendersi troppo sul serio"; sullo stile di scrittura della sua Storia dei Romani, per esempio, De Sanctis replica nella lettera del 30 VIII 1906: "Ciò che è stato concepito in un modo non deve essere per così dire travasato in uno stile adatto a modo di concepire affatto diverso. Si rischierebbe di vestire con un incongruo palamidone uso Giolitti un giovane agile, snello e nervoso. Tuttavia quando quel giovane per la smania di camminare presto, ridà a saltellare, lo si può richiamare all'ardire ${ }^{1}$ e fargli riprendere un passo più adatto ad una persona ammodo. Del franco tuo giudizio a ogni modo ti ringrazio vivamente. Le lodi degli amici son pericolose, non i biasimi » (p. $60 ;$ p. 13 dell'Introduzione).

3 Una prima parte del carteggio è percorsa da un Leitmotiv costante, inerente alla vita privata di Fraccaroli, per cui l'amico e collega De Sanctis si prodiga moltissimo : la complicata causa di separazione di Fraccaroli dalla moglie Isabella Quaglio Rezzonico. La vertenza legale della separazione ha per sede Torino, i suoi dicasteri, l'Ateneo stesso (a causa dei pignoramenti di stipendio, della domanda di aspettativa e della lettera di dimissioni dall'insegnamento da parte di Fraccaroli: tutte conseguenze della controversia coniugale, rintracciabili fino alla lettera XXVII [27]). De Sanctis dà ragguagli anche di altre notizie, a proposito di nuove pubblicazioni, polemiche accademiche, carriera di allievi comuni e lavoro dei colleghi ; ma la causa civile e i suoi risvolti appaiono l'occasione prima di questo gruppo di lettere, soprattutto perché De Sanctis deve informare Fraccaroli sui progressi (o sulle stagnazioni) della vicenda, sulla scarsa combattività dei suoi avvocati (contro quelli, assai più agguerriti, della moglie), chiedere istruzioni a Fraccaroli su come rendersi utile e fare da intermediario tra i due coniugi in rotta. Ma, al di là delle questioni spicciole e pragmatiche poste, avviate e in parte anche risolte da De Sanctis per conto di Fraccaroli, non va dimenticato un discrimine cronologico fondamentale per la storia culturale dei due protagonisti e di tutta la scienza dell'antichità del periodo : nel 1903 era stato pubblicato a Torino il libro più importante della produzione di Fraccaroli, L'irrazionale nella letteratura. Con quest'opera Fraccaroli tentava di sistematizzare i numerosi spunti anti-filologici degli anni precedenti, soprattutto in polemica nei confronti di Girolamo Vitelli e della sua scuola (in particolare Nicola Festa). "Gracile e semplicistico l'impianto "filosofico" del lavoro - non la ragione, bensì l'irrazionale è l'elemento costante e necessario dell'arte, onde a questa ci si potrà accostare solo per via irrazionale. Filologia e critica razionale vengono dunque bandite : perché "la ragione smorza l'amore", mentre "l'arte vuole amanti e non medici" (p.8). L'asserto è illustrato con esempi tratti soprattutto da Omero, per cui l'opera, in nome della "libertà" poetica, viene a negare ogni legittimità alla cosiddetta "questione omerica" » (così riassumeva gli intenti del libro Enzo Degani, La filologia greca nel secolo Xx [Italia], ora in Filologia e storia. Scritti di E. D., II, HildesheimZürich-New York 2004, p. 1087). La reazione di De Sanctis alla lettura del libro di così radicale assunto $\mathrm{fu}$, come al solito, temperata dall'equilibrio, dal rispetto, dalla pacatezza. Con la schiettezza rimeditata sull'entità delle proprie convinzioni (vale a 
dire : a lettura e recensione del volume ultimata), De Sanctis preannuncia a Fraccaroli un motivo di dissenso nella lettera del 25 I 1904 : « [...] avrò tra breve gli estratti della mia recensione del tuo libro. Come sai, difendo a spada tratta la critica omerica di Lachmann e successori contro di te. Spero che non me ne vorrai male» (p. 45 : si tratta di un passaggio giustamente posto in rilievo e analizzato da Guglielmo, insieme ad altri, alle p. 14-16 dell'Introduzione). La confutazione della teoria unitaria sulla composizione dei poemi omerici (sostenuta da Fraccaroli nel libro del 1903) anima l'articolo di De Sanctis, L'irrazionale nell'Iliade, « RFIC » 32, 1904, p. 41-57 ; ma anni più tardi, nella lettera del 18 III 1915, De Sanctis confessa a Fraccaroli che se la sua propria impostazione didattica, il suo approccio ai testi, all'esegesi, all'insegnamento, insomma se la sua scuola « è meno imperfetta di quel che potrebbe essere, si deve in parte alla meditazione appunto di quel tuo libro» (p. 122 s.; p. 15 dell'Introduzione). Di tale signorilità, pur nella polemica - per riprendere un tema già ricordato -, è esempio anche il Fraccaroli della replica alla recensione di De Sanctis, nell'articolo L'irrazionale e la critica omerica (" RFIC » 33, 1905, p. 273-291) : «La polemica utile è quella che si fa con gli amici. [...] quella con gli amici, e amici che si amano, che si stimano e che si rispettano, può diventare un piacere eletto, e può essere veramente utile e a chi discute e a chi ascolta la discussione » (p. 2 dell'Introduzione).

4 Alla triste vicenda coniugale di Fraccaroli è legato il primo documento dell'Appendice; se la raccolta di lettere pubblicata nel volume riguarda la scrittura di De Sanctis, i testi dell'Appendice sono piuttosto inerenti all'opera di Fraccaroli, per una sorta di bilanciamento tra i documenti riconducibili all'uno e all'altro studioso. A firma di Fraccaroli stesso è una Lettera aperta a S.E. il Ministro dell'struzione Pubblica, datata Milano, 19 II 1907 (p.131-137), in cui giustifica la sua impossibilità a riprendere l'insegnamento presso l'Università di Torino, dopo due anni di aspettativa per motivi familiari; il tono della lettera è apertamente ostile nei confronti della moglie, considerata pervicace responsabile delle sciagure professionali di chi scrive, delle estenuanti peripezie giudiziarie (fanno da contraltare tutti i suggerimenti e le premure nelle lettere di De Sanctis), e del dissesto economico conseguente all'interruzione dell'insegnamento. A causa del pignoramento di una frazione di stipendio, comminato a Fraccaroli da un tribunale che ha accolto alcune istanze della moglie nella causa di separazione, Fraccaroli decide di non riprendere più servizio di professore, "preferendo il nulla al poco» (come commenta dispiaciuto lo stesso De Sanctis, p. 67). La scelta della curatrice di ripubblicare questo documento (nel complesso imbarazzante, quando non sgradevole nei contenuti e negli intenti polemici - pur dalla parte della ragione, per quanto è dato capire) va considerata senza dubbio opportuna e utile; soltanto dopo aver letto questo memoriale ufficiale si comprende infatti l'amarezza di De Sanctis, nella lettera datata 23 II 1907, che in tutta franchezza considera la missiva di Fraccaroli « inutile, imprudente, illogica e persino (bisogna pur dire la verità) immorale» (p.66). Ma ha il sopravvento il dispiacere dell'amico, sinceramente addolorato di perdere in Fraccaroli un illustre collega, allorché prorompe: "Con la energia morale che tu hai, se tu qualche volta, solo qualche rara volta, avessi ascoltato i consigli degli amici, come si sarebbe potuto rimediare bene alle disgrazie che ti hanno colpito !» (p. 67).

5 Gli altri tre documenti dell'Appendice vertono su una schermaglia propriamente scientifica, originata dalla pubblicazione dei due volumi di traduzioni di Fraccaroli, I lirici greci (poesia melica), apparsi nella collana « Il pensiero greco » delle edizioni Fratelli 
Bocca di Torino nel 1913. Nel primo numero (1913) di « La Nuova Cultura », periodico appena ricostituito da Giuseppe Antonio Borgese (in sostituzione e a prosecuzione del precedente «La Cultura ») Gaetano Munno recensisce con acredine le traduzioni di Fraccaroli. Marcella Guglielmo, che ha inserito questo testo nell'Appendice (p. 138-145), così lo sintetizza : "Le critiche mossegli sono severe : mordaci nella forma, che nulla lascia a espressioni anche solo apparentemente diplomatiche, e inflessibili nei giudizi, che sottolineano l'oscura ambiguità delle traduzioni poetiche, con tanto di segnalazione di versi a suo avviso poco efficaci o maldestri » (Introduzione, p. 23). La pubblicazione di questa recensione crea uno scandalo, oltre a suscitare l'indignazione di Fraccaroli e del collega De Sanctis, anche perché «La Nuova Cultura » è periodico edito dai Fratelli Bocca, lo stesso editore dei Lirici di Fraccaroli. Le confutazioni di Munno, a quanto pare, sono tutte infondate ; ma è bene lasciare la parola all'epistolario, per rendersi conto ancora una volta di come la trasparenza e la sincerità costituiscano il principale, reciproco atteggiamento dei protagonisti : in generale, e poi riguardo a un passo specifico (Bacchil. 5, 66 s. Snell-Maehler), scrive De Sanctis a Fraccaroli il 2 X 1913 : «Non ho parole per esprimere lo schifo che suscita in me quella sozza prosa, materiata d'impostura e d'ignoranza. [...] Gli appunti mossi alla tua versione sono tutti falsificazioni, futilità o spropositi del critico ; tutti, salvo uno : quello dell' ó vò $\pi \rho \tilde{\omega} v \alpha \varsigma$

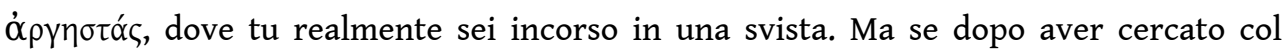
microscopio, in un volume di quasi seicento pagine il tuo detrattore non è riuscito a rilevare che una svista, vuol dire che la tua versione è ottima » (p. 96). Fraccaroli aveva tradotto il passo di Bacchilide in questione «Simili a foglie pallide / Che agita il vento sulle rupi e i clivi / Erbosi d'Ida »; Munno contestava la convinzione « che l'epiteto ó $\rho$

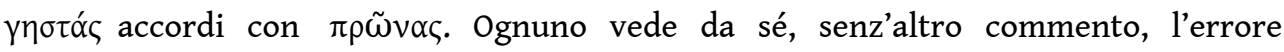
madornale !...» (p. 139). Risponde Fraccaroli a De Sanctis il 4 X 1913 : « Tu mi dici che

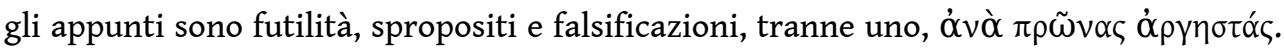
Ora ti prego di leggere la nota a quel luogo (p. 454), e vedrai che è una falsificazione anche quello» (p. 97 n. 292). De Sanctis il 7 dello stesso mese : «Leggendo, vedrai tu

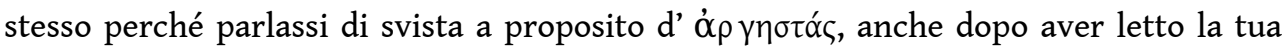

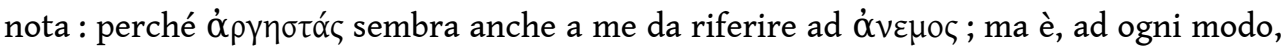

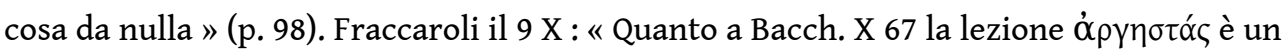

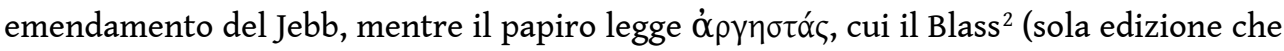

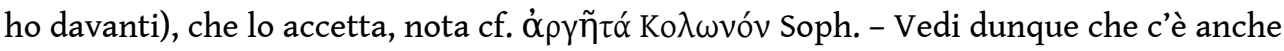
qui un'impostura » (p. 98 n. 298 ; il rimando di Blass è all'Edipo a Colono, v. 670). Il 2 XI, in calce alla lettera, dopo la firma, De Sanctis si convince e conclude la breve

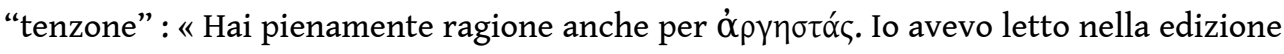

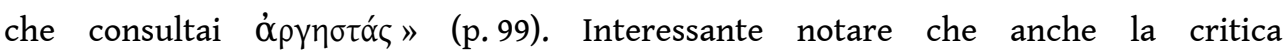
contemporanea sottolinea l'insidia editoriale ed esegetica del passo, poiché in una nota d'apparato dell'edizione di riferimento (Bacchylidis Carmina cum fragmentis, post B. Snell ed. H. Maehler, Lipsiae 1970) si mette in guardia dal possibile fraintendimento

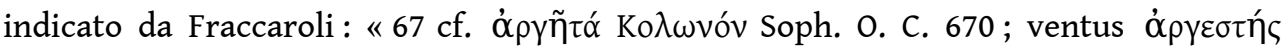

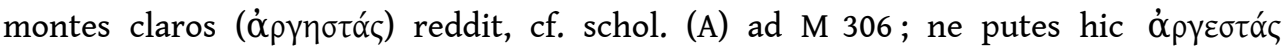
scribendum esse » (p. 17).

$6 \mathrm{Ma}$, pur spiegatisi tra loro i due cattedratici, la polemica non finisce certo a questo punto. Nell'ultimo fascicolo del 1913 della « Nuova Cultura » Fraccaroli provvede a rispondere alla feroce recensione con una lettera inviata all'editore, Avvocato Giuseppe Bocca (che Guglielmo trascrive alle p.146-155 dell'Appendice), in cui sono ricordate 
altrui accuse di plagio e di scorrettezza metodologica contro Munno, a proposito di precedenti lavori. Per quanto riguarda il passo di Bacchilide, oltre a tutti gli altri, Fraccaroli ripete distesamente quanto già anticipato a De Sanctis, ma con tono arguto $\mathrm{e}$

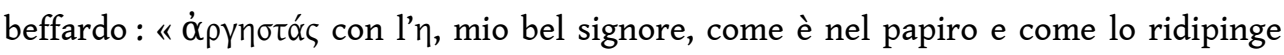
anche Lei, per sua buona regola, concorda con $\pi \rho \tilde{\omega} v \alpha \varsigma$, proprio davvero, come ognuno

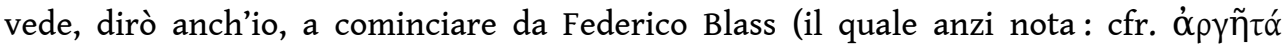
Ko $\lambda \omega v o ́ v$, Soph.) per terminare con l'ultimo ragazzetto che ha meritato la promozione

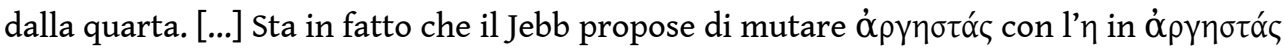
con l' $\varepsilon$, noto epiteto del vento, che si scrive appunto con l' $\varepsilon$, una congettura arbitraria e inutile affatto » (p. 149). Epilogo (per nulla riconciliante) : l'anno dopo Gaetano Munno pubblica un opuscoletto a parte, Lirici greci e traduttori italici (Risposta al Professore Fraccaroli), Roma 1914 (ora a conclusione dell'Appendice, p. 156-174). E senza retrocedere di un passo, sul locus conclamatus di Bacchilide scrive tra l'altro : « Il Fraccaroli riporta la citazione del Blass - $\alpha$ $\rho \gamma \tilde{\eta} \tau \alpha ́$ Ko $\lambda \omega v o ́ v$ (Sofocle, Edipo a Colono, 670), con la quale, neppure a farlo apposta, si getta la zappa sui piedi. L' $\alpha \rho \gamma \tilde{\eta} \tau \alpha$ in Sofocle significa appunto biancheggiante; lo notarono già gli scoliasti, che ammiravano nel coro sofocleo il voluto contrasto fra il candido cocuzzolo e le verdi convalli. Candido dunque ! ed è

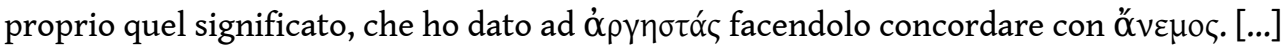
cioè candens nel senso di acceso (parliamo pure d'incandescenza anche in italiano; e le escandescenze del sig. Fraccaroli pure sono di quella famiglia)» (p. 160). Ma, per dare ragione definitiva a Fraccaroli contro le pretese di Munno, sarà utile richiamare almeno due traduzioni contemporanee del passo di Bacchilide, una italiana e una francese : "quante sono le foglie / che il vento sulle balze assolate / dell'Ida tra le greggi disperde » (R. Sevieri, Milano 2007), e «telles les feuilles qu'agite le vent, par les blancs promontoires de l'Ida où paissant les brebis » (J. Duchemin e L. Bardollet, Paris 1993, per l'edizione di J. Irigoin nella « Collection des Universités de France »).

7 E per un bilancio conclusivo e complessivo di tutti i documenti acclusi nel bel libro di Marcella Guglielmo, dopo queste poche esemplificazioni valgano le parole della stessa curatrice: "Cronaca privata dunque, e storia epicorica, ma soprattutto critica letteraria e momenti di vita intellettuale del primo ventennio del Novecento traspaiono da questo carteggio, attraverso le riflessioni e le reazioni di due tra i protagonisti, tanto diversi per età e per formazione, ma vicini per il legame di amicizia e di rispetto che li univa » (p. 27).

8 Concludono il volume una Bibliografia strutturata in più sezioni (p. 177-184) e un utile Indice dei nomi (p. 187-192).

\section{NOTE}

1. All'ardire: forse bisogna leggere all'ordine. 


\section{AUTORE}

\section{MICHELE CURNIS}

Università degli Studi di Torino

bellerofonte@gmx.net 